\title{
Functional Impact of Dendritic Branch-Point Morphology
}

\author{
Michele Ferrante, ${ }^{1}$ Michele Migliore,${ }^{2}$ and Giorgio A. Ascoli ${ }^{1}$ \\ ${ }^{1}$ Center for Neural Informatics, Structures, and Plasticity, and Molecular Neuroscience Department, Krasnow Institute for Advanced Study, George Mason \\ University, Fairfax, Virginia 22030, and 2Institute of Biophysics, National Research Council, I-90146 Palermo, Italy
}

Cortical pyramidal cells store multiple features of complex synaptic input in individual dendritic branches and independently regulate the coupling between dendritic and somatic spikes. Branch points in apical trees exhibit wide ranges of sizes and shapes, and the large diameter ratio between trunk and oblique dendrites exacerbates impedance mismatch. The morphological diversity of dendritic bifurcations could thus locally tune neuronal excitability and signal integration. However, these aspects have never been investigated. Here, we first quantified the morphological variability of branch points from two-photon images of rat CA1 pyramidal neurons. We then investigated the geometrical features affecting spike initiation, propagation, and timing with a computational model validated by glutamate uncaging experiments. The results suggest that even subtle membrane readjustments at branch points could drastically alter the ability of synaptic input to generate, propagate, and time action potentials.

\section{Introduction}

Dendritic integration and compartmentalization in hippocampal pyramidal neurons are believed to play a direct role in spatial information processing (Nolan et al., 2004; Morita 2008). Local distribution of active properties (Losonczy et al., 2008), dendrite/trunk diameter ratio (Migliore et al., 2005), and synaptic location ( $\mathrm{Li}$ and Ascoli, 2006) are all key determinants of dendritic $\mathrm{Na}^{+}$spike (d-spike) initiation and propagation (Golding and Spruston, 1998; Gasparini et al., 2004). At the same time, a rich repertoire of biological mechanisms can dynamically change dendritic computation in response to cell activity (Johnston and Narayanan, 2008). Synaptic (Caporale and Dan, 2008), intrinsic (Turrigiano et al., 1994), and structural (Bourne and Harris, 2008) plasticity, respectively, regulate the strength of input signals, ionic channel expression (and/or biophysical properties), and local morphology. Moreover, activity-dependent structural and functional changes may co-occur, as in synapses changing their shape and size when potentiating or depressing.

CA1 pyramidal neurons are characterized by a thick apical trunk from which arise spiny oblique dendrites. Although these dendrites are thin $(\sim 0.5 \mu \mathrm{m}$ in diameter $)$ and represent only $\sim 35 \%$ of the dendritic wiring, they receive approximately

Received July 21, 2012; revised Dec. 4, 2012; accepted Dec. 5, 2012.

Author contributions: M.F., M.M., and G.A.A. designed research; M.F. performed research; M.F. contributed unpublished reagents/analytic tools; M.F. analyzed data; M.F., M.M., and G.A.A. wrote the paper.

This work was supported by National Institutes of Health Grant R01 NS39600 and Office of Naval Research Grant MURI 14-10-1-0198 (G.A.A.). We are extremely grateful to Drs. Judit K. Makara and Jeffrey C. Magee (Janelia Farm Research Campus, Howard Hughes Medical Institute, Ashburn, VA) for supplying the confocal image stacks, performing glutamate uncaging experiments, and providing critical feedback on previous versions of this manuscript. Dr. Stephen Smith (Stanford University, Palo Alto, CA) is also warmly acknowledged for sharing neuronal microscopy movies.

The authors declare no competing financial interests.

Correspondence should be addressed to Giorgio A. Ascoli at the above address. E-mail: ascoli@gmu.edu.

M. Ferrante's present address: Center for Memory and Brain, Department of Psychology, Boston University, Boston, MA 02215.

DOI:10.1523/JNEUROSCI.3495-12.2013

Copyright $\odot 2013$ the authors $\quad 0270-6474 / 13 / 332156-10 \$ 15.00 / 0$ half of the total excitatory inputs (Megías et al., 2001) and may act as independent computational units within the dendritic tree (Poirazi and Mel, 2001; Losonczy et al., 2008). With high local input impedance, high-impedance mismatch with the main trunk, and high active excitability, obliques are ideally suited to perform local computation, while still contributing to the overall neuronal output (Rabinowitch and Segev, 2006; Katz et al., 2009). The influence of dendritic morphology on the input/output relationship has been investigated extensively in cortical and hippocampal neurons (Mainen and Sejnowski, 1996; Krichmar et al., 2002; Schaefer et al., 2003; Komendantov and Ascoli, 2009). In contrast, the morphological diversity and functional role of the bifurcation between oblique dendrites and the apical trunk has been essentially ignored and experimentally unexplored.

This work investigates the role of branch-point morphology in determining the minimum synaptic stimulation required to generate either a d-spike in an oblique branch or a somatic action potential (AP). In CA1 pyramidal neurons, dendritic compartmentalization can be regulated by active properties, such as potassium channels (Cai et al., 2004). Although the effects of synaptic and intrinsic plasticity on spike initiation and propagation have been investigated extensively (Canepari et al., 2007; Remy et al., 2009), the functional consequences of morphological changes at dendritic bifurcations are unknown and difficult to explore experimentally. We observed that branch points in the apical dendrites of CA1 pyramidal neurons exhibit considerable structural variability, which might result from activity-dependent regulation. Our results suggest that minor (possibly dynamic) alterations of a small patch of membrane at dendritic bifurcations might have major and opposite effects on the probabilities to initiate and propagate a spike, as well as on its onset and temporal reliability. Much as structural plasticity of dendritic spines can control electric compartmentalization and synaptic communication (Grunditz et al., 2008; Fu and Zuo, 2011), bifurcation morphol- 
ogy might be a powerful regulator of branch excitability and coupling.

\section{Materials and Methods}

Hippocampal slice preparation and patch-clamp recordings. Image acquisition and glutamate uncaging slice experiments were performed by Drs. Judit K. Makara and Jeffrey C. Magee at the Janelia Farm Research Campus of the Howard Hughes Medical Institute (Ashburn, VA); these data have then been kindly ceded to the authors. Adult (8- to 12-week-old) male Sprague Dawley rats were used to prepare transverse slices (400 $\mu \mathrm{m}$ ) from the middle to dorsal part of the hippocampus similarly to previous descriptions (Magee, 1998), according to methods approved by the Janelia Farm Institutional Animal Care and Use Committee. Experiments were performed at $33-35^{\circ} \mathrm{C}$ in ACSF containing the following (in m): $125 \mathrm{NaCl}, 3 \mathrm{KCl}, 25 \mathrm{NaHCO}_{3}, 1.25 \mathrm{NaH}_{2} \mathrm{PO}_{4}, 1.3 \mathrm{CaCl}_{2}, 1 \mathrm{MgCl}_{2}$, 25 glucose, $3 \mathrm{Na}$-pyruvate, and 1 ascorbic acid (saturated with $95 \% \mathrm{O}_{2}$ and $5 \% \mathrm{CO}_{2}$ ). Cells were visualized using an Olympus BX-61 epifluorescent microscope equipped with differential interference contrast optics under infrared illumination and a water-immersion lens $(60 \times, 0.9 \mathrm{nu}-$ merical aperture; Olympus). Current-clamp whole-cell recordings from the somata of hippocampal CA1 pyramidal neurons were performed using a Dagan BVC-700 amplifier in the active "bridge" mode, filtered at $3 \mathrm{kHz}$ and digitized at $50 \mathrm{kHz}$. Patch pipettes (2-6 M $\Omega$ ) were filled with a solution containing $100 \mu \mathrm{M}$ Alexa Fluor 488 and the following (in $\mathrm{mm}$ ): 120 K-gluconate, $20 \mathrm{KCl}, 10 \mathrm{HEPES}, 4 \mathrm{NaCl}, 4 \mathrm{Mg}_{2} \mathrm{ATP}, 0.3 \mathrm{Tris}_{2} \mathrm{GTP}$, and 14 phosphocreatine, $\mathrm{pH} 7.25$. Series resistance was $<20 \mathrm{M} \Omega$. All neurons had resting membrane potentials between -58 and $-65 \mathrm{mV}$. The membrane potential was kept slightly hyperpolarized (approximately -65 to $-70 \mathrm{mV}$ ), to better isolate local $\mathrm{Na}^{+}$spikes from axosomatic APs.

Two-photon imaging and uncaging. A dual galvanometer-based twophoton scanning system (Prairie Technologies) was used to obtain fluorescent images of Alexa Fluor 488-loaded neurons and to uncage glutamate at individual dendritic spines (Losonczy and Magee, 2006; Losonczy et al., 2008; Makara et al., 2009). Two ultrafast pulsed laser beams (Chameleon Ultra II; Coherent) were used, one at $920 \mathrm{~nm}$ for imaging Alexa Fluor 488 (Invitrogen) and one at $720 \mathrm{~nm}$ to photolyze 4-methoxy-7-nitroindolinyl-caged L-glutamate (MNI-glutamate) (Tocris Cookson; $10 \mathrm{~mm}$ applied through a pipette above the slice). Laser beam intensity was independently controlled with electro-optical modulators (model 350-50; Conoptics).

All uncaging experiments were performed in proximal apical firstorder oblique dendrites, branching off from the soma or from the apical trunk within $60 \mu \mathrm{m}$ from the soma. Local dendritic $\mathrm{Na}^{+}$spikes were evoked by synchronous uncaging of MNI-glutamate at a spatially close cluster of 4-12 visually identified spines $(0.2 \mathrm{~ms}$ uncaging duration with $0.1 \mathrm{~ms}$ intervals between exposures for scanning mirror repositioning). Generation of a local spike could be unequivocally identified by the appearance of a fast spikelet on the rising phase of the compound glutamate-uncaging-induced EPSP (gluEPSP) and a corresponding peak on the $\mathrm{dV} / \mathrm{dt}$ (instantaneous rate of voltage change over time) of the voltage signal (Losonczy and Magee, 2006; Losonczy et al., 2008; Makara et al., 2009). To determine spike threshold (as measured at the soma) at a given dendritic location, the uncaging laser power was adjusted to the lowest level that was sufficient to evoke the spike with synchronous uncaging, and the same power was used to record the individual spine responses (with $210 \mathrm{~ms}$ interval) in the same experimental session (see Fig. 2C-E). Spike threshold was calculated as the arithmetic sum of the individual gluEPSPs.

Morphological analyses. Morphological measurements were extracted with NIH ImageJ from high-resolution ( $>5 \mathrm{pixel} / \mu \mathrm{m}$ on $x / y$ ) image stacks of CA1 pyramidal neurons. Branch-point tapering measures were collected from 2D maximum intensity for individual image stacks, selecting the branches that were quasi-parallel to the $x / y$ plane for highest resolution. Four morphological parameters were recorded for each of the 171 branches of the 69 CA1 pyramidal neurons: (1) $d_{\text {trunk }}$ (diameter of main trunk); (2) $d_{\text {dend }}$ (diameter of the dendrite); (3) lc (length of the tapering cylinder); and (4) $d_{\text {taper }}$ (diameter of the tapering region at the trunk). From these parameters, we computed the branch-point surface (surf) as the lateral surface area of the circular conical frustum using the following formula:

$$
\operatorname{surf}=\pi\left[\left(d_{\text {taper }}+d_{\text {dend }}\right) / 2\right] \operatorname{sqrt}\left\{\left[\left(d_{\text {taper }}-d_{\text {dend }}\right) / 2\right]^{2}+1 \mathrm{c}^{2}\right\} .
$$

Neurons were imaged either before ( $n=52$ neurons) or after $(n=17)$ uncaging. None of the measured distributions $\left(d_{\text {dend }}, d_{\text {trunk }}, d_{\text {taper }}\right.$, and lc) revealed any statistical difference between the two conditions $(p>0.2$ in all cases), indicating that glutamate uncaging did not significantly affect local branch morphology. To minimize the possible bias introduced by subjective measurements, a different coauthor blindly reextracted and independently analyzed the morphological features from a subsample of the branches $(n=30)$. The two sets of measurements for each morphological feature were highly correlated $\left(R^{2}>0.87\right)$ and not significantly different $(p>0.1)$. The average difference was $<6 \%$ for every parameter, and the discrepancy between any two individual measurements never exceeded $18 \%$.

Computational model. All simulations were implemented and run in NEURON (Hines and Carnevale, 1997) version 7.2 with variable time step, on a 64-bit Pentium quad-core Dell XPS laptop under Windows 7. Model and simulation files are available for public download under the ModelDB section (accession number 146509) of the Senselab database (senselab.yale.med.edu/ModelDB). Because of the variable resolution of available digital reconstructions of dendritic morphology (Scorcioni et al., 2004) and for the sake of parsimony and standardization, we implemented a simplified T-shaped multicompartmental model. Unless otherwise noted, the model used for all simulations consisted of three segments: (1) a $240-\mu \mathrm{m}$-long trunk; (2) a $120-\mu \mathrm{m}$-long oblique dendrite; and (3) a bifurcation tapering segment stemming off the middle of the trunk and connecting one end of the oblique dendrite. All simulations used the morphological parameters measured from the image stacks.

The same active and passive properties previously described and validated (Migliore et al., 2005) were used in all cases. Briefly, $\tau_{\mathrm{m}}=28 \mathrm{~ms}$, $R_{\mathrm{m}}=28 \mathrm{k} \Omega-\mathrm{cm}^{2}, R_{\mathrm{a}}=150 \Omega-\mathrm{cm}, I_{\mathrm{Na}}$ and $I_{\mathrm{Kdr}}$ uniformly distributed throughout the membrane surface (with peak conductances $g_{\mathrm{Na}}=0.25$ $\mathrm{nS} / \mu \mathrm{m}^{2}$ and $\left.g_{\mathrm{Kdr}}=0.1 \mathrm{nS} / \mu \mathrm{m}^{2}\right) ; I_{h}$ and $I_{\mathrm{KA}}$ linearly increased with distance, dist, as $g_{\mathrm{KA}}=0.3 \times\left(1+\right.$ dist/100) and $g_{h}=0.0005 \times(1+$ 3dist/100) (Hoffman et al., 1997; Magee, 1998). AMPA-type excitatory synapses $(0 \mathrm{mV}$ reversal potential) were implemented using the NEURON double-exponential function $\operatorname{Exp} 2 \operatorname{Syn}()$, with rise $(0.5 \mathrm{~ms})$ and decay ( $3 \mathrm{~ms}$ ) times consistent with experimental measurements (Andrasfalvy and Magee 2001).

To validate the model against two-photon uncaging experiments, in a subset of simulations, we stochastically chose three synaptic locations on the dendrite between 10 and $75 \mu \mathrm{m}$ from the branch point for each set of morphometric parameters, positioning the recording electrode on the trunk at a random distance between 0 and $100 \mu \mathrm{m}$ from the branch point. For each synaptic location, we increased the synaptic strength to elicit a d-spike while recording the trunk depolarization. For all other simulations, the model included two recording electrodes: one at the location of the synaptic input (at $38 \mu \mathrm{m}$ from the branch point unless otherwise stated) and one in the trunk at $84 \mu \mathrm{m}$ from the branch point. The stimulation protocol consisted of progressively increasing for each branch-point configuration the strength of the synapse to record an EPSP, a d-spike, or an AP (and its onset). $\theta_{\text {d-spike }}$ and $\theta_{\mathrm{AP}}$ were defined as the synaptic input strength necessary to evoke either a d-spike or an AP, respectively.

\section{Results}

\section{Branch-point morphology is remarkably diverse and heavily} affects dendritic spike initiation and propagation

To characterize branch-point morphology in CA1 pyramidal neurons, we identified 171 bifurcations between oblique branches and the main apical trunk from two-photon confocal microscopy image stacks of 69 neurons, 18 of which were used in a previous study (Makara et al., 2009). For each of these, we measured and analyzed four parameters (Fig. 1A): (1) $d_{\text {trunk }} ;(2)$ 
$d_{\text {dend }}$; (3) $d_{\text {taper; }}$; and (4) lc (all defined in Materials and Methods). All parameters, as well as surf and the $d_{\text {dend }} / d_{\text {trunk }}$ ratio, showed a considerably broad range of values (Fig. $1 B$ ) and several significant correlations ( $d_{\text {dend }}$ vs lc, $r=0.57, p<0.001$; $d_{\text {trunk }}$ vs surf, $r=0.52, p<0.001 ; d_{\text {trunk }}$ vs lc, $r=0.48, p<0.01 ; d_{\text {dend }}$ vs $d_{\text {trunk }}, r=$ $0.41, p<0.05$; $r$ values are Pearson's product moment correlation coefficients).

We quantified the morphometric variability of each feature by the coefficients of variation $(\mathrm{CVs})$ across all branch points pooled together (overall CV) as well as between and within neurons. The betweenneuron $\mathrm{CV}$ was computed on the averages of the appropriate feature over all branches of each neuron. The withinneuron $\mathrm{CV}$ was the average of the corresponding CVs for each feature computed for the set of branch points within every neuron. This latter metric was only computed over the 33 neurons with at least three characterized branches. The least variable features were $d_{\text {dend }}$ and $d_{\text {trunk }}$ (overall CV, 28.2 and $28.3 \%$, respectively). Interestingly, $d_{\text {dend }}$ displayed greater variability within than between neurons (23.7 vs $19.2 \%$ ), whereas the opposite held for $d_{\text {trunk }}$ (within CV, 17.4\%; between CV, 24.3\%). In contrast, surf was the most variable feature $(\mathrm{CV}$ values of $86.1,62.5$, and $58.4 \%$, respectively, for overall, between, and within neurons).

Propagation of $\mathrm{d}$-spikes from the oblique onto the trunk is mainly modulated by $d_{\text {dend }} / d_{\text {trunk }}$ via impedance mismatch (Migliore et al., 2005), and the observed morphological diversity might imply a corresponding functional diversity in local computation. To investigate the effect of different, experimentally measured, branch-point morphologies on the coupling between d-spikes and somatic APs, we used a computational model to determine the stimulation thresholds necessary for local spike initiation and propagation. First, to demonstrate the main effect, using the average values of $d_{\text {dend }}, d_{\text {trunk }}, \mathrm{lc}$, and $d_{\text {taper }}$, we found the minimum peak synaptic conductances eliciting a local d-spike and a propagated $\mathrm{AP}$ in the trunk $\left(\theta_{\mathrm{d} \text {-spike }}\right.$ and $\theta_{\mathrm{AP}}$, respectively) as a function of the input location. A synapse on the oblique branch is considered fully coupled to the trunk if the stimulus thresholds to generate a d-spike and an AP are the same, i.e., any d-spike would trigger an AP. Conversely, a synapse is highly uncoupled if the two thresholds are very different, i.e., a broad range of input strengths would generate a d-spike but not an AP. The results suggested that synapses are fully coupled relatively near $(<40 \mu \mathrm{m})$ the bifurcation point,

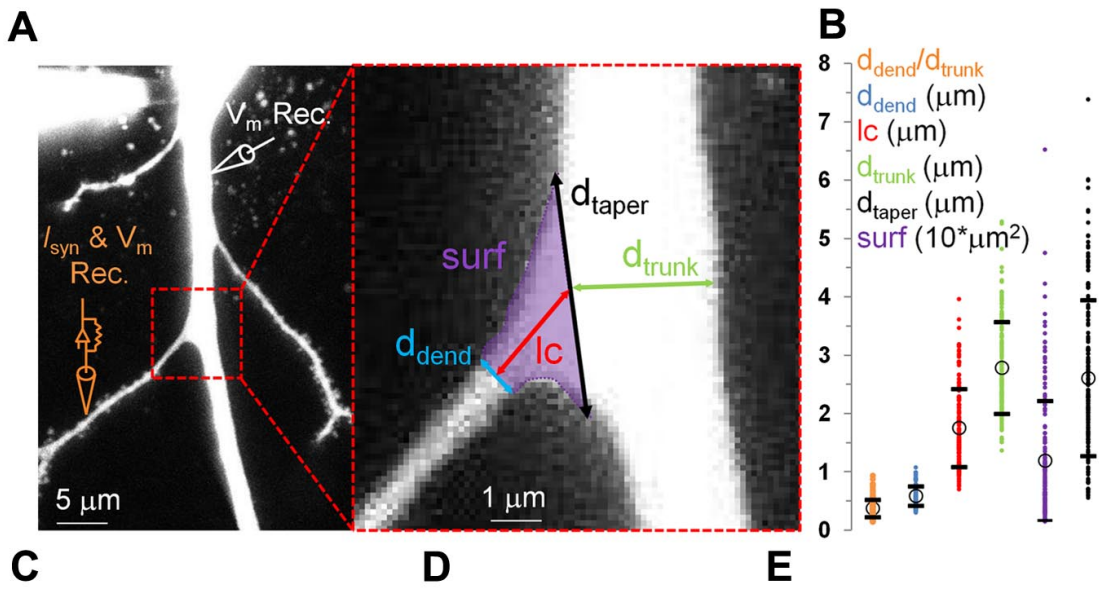

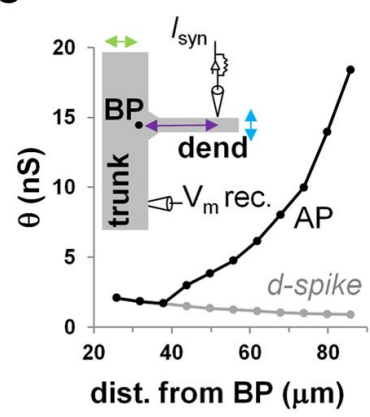

$\mathbf{F}$

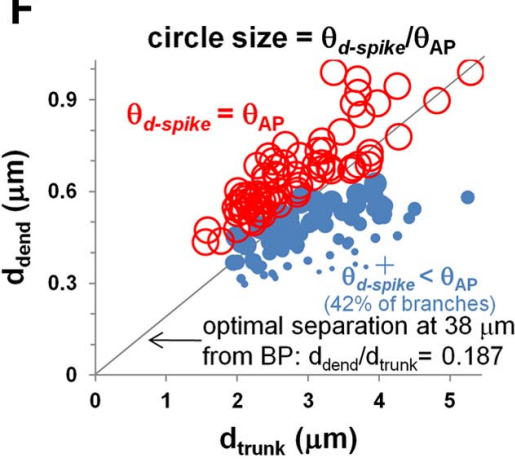

$\mathbf{G}$

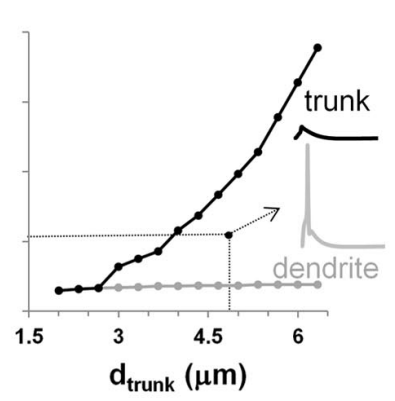

Figure 1. Branch-point (BP) morphology affects the thresholds for dendritic and somatic spike generation. A, Left, Representative confocal image illustrating the simulation setup (orange, stimulating and recording electrode; white, recording electrode). Right, Expanded view of the bifurcation between the main trunk and the oblique branch, with the measured morphometric parameters: $d_{\text {dend }} d_{\text {trunk' }}$ surf, and Ic. $\boldsymbol{B}$, Parameter distributions for the 171 branches measured from image stacks; circles and horizontal lines mark averages and SDs, respectively. $\mathbf{C}-\boldsymbol{E}$, Stimulation threshold $(\theta)$ to generate a local d-spike (gray line) or an AP in the trunk (black line) in the computational model, as a function of the distance of the input from the branch point $(\boldsymbol{C})$, the trunk diameter $(\boldsymbol{D})$, the diameter of the oblique dendrite $(\boldsymbol{E})$, and the oblique/trunk diameter ratio (inset in $\boldsymbol{E}$ ). The inset in $\boldsymbol{C}$ depicts a schematic of the computational model in which purple, green, and blue arrows show the manipulated parameters in $\boldsymbol{C}, \boldsymbol{D}$, and $\boldsymbol{E}$, respectively. The inset in $\boldsymbol{D}$ shows dendritic and trunk voltage traces during a $5 \mathrm{nS}$ synaptic stimulation at $38 \mu \mathrm{m}$ from the branch point. Except for the independent variables in each of the main panels, average branch morphometric values $(\boldsymbol{B})$ and a $38 \mu \mathrm{m}$ synaptic distance from the branch point were used for all parameters. In contrast, the inset in $\boldsymbol{E}$ used the original values extracted from the 171 bifurcations. $F$, Linear discriminant analysis identifies, for a specific synaptic distance from the branch point $(38 \mu \mathrm{m})$, the optimal $d_{\text {dend }} / d_{\text {trunk }}$ diameter ratio allowing thresholds separation. Red and blue circles show synapses with coupled $\left(\theta_{\mathrm{d}-\text { spike }}\right.$ $\left.=\theta_{\mathrm{AP}}\right)$ and compartmentalized $\left(\theta_{\mathrm{d}-\text { spike }}<\theta_{\mathrm{AP}}\right)$ thresholds, respectively. Symbol size represents $\theta_{\mathrm{d}-\text { spike }} / \theta_{\mathrm{AP}}$ threshold ratio, and the + symbol indicates the single case in which the d-spike never propagated into the trunk, even with a stimulation of $40 \mathrm{nS}$. $\mathbf{G}$, Optimal $d_{\text {dend }} / d_{\text {trunk }}$ values for threshold separation as a function of stimulation distance from the branch point. Red area depicts coupled synapses, blue gradient shows different degrees of thresholds separation, with darker shade indicating greater compartmentalization. The black dot corresponds to data shown in $\boldsymbol{F}$. $\boldsymbol{H}$, Black line, Average level of branch compartmentalization for the compartmentalized branches as a function of the synaptic distance from the branch point. Percentages, Amount of compartmentalized branches; dotted lines, average \pm SD level of branch compartmentalization. but the d-spike and AP thresholds dramatically diverge farther on the oblique dendrite (Fig. 1C). More specifically, the distance of the input from the branch point had opposite effects, respectively, decreasing $\theta_{\mathrm{d}-\text { spike }}$ and increasing $\theta_{\mathrm{AP}}$. 
A
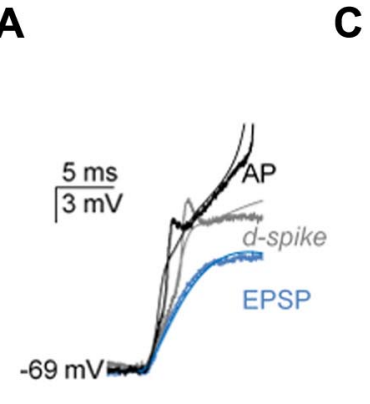

B

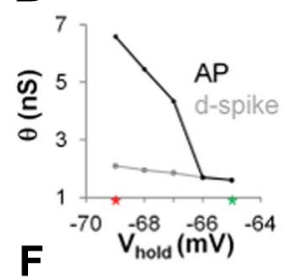

D
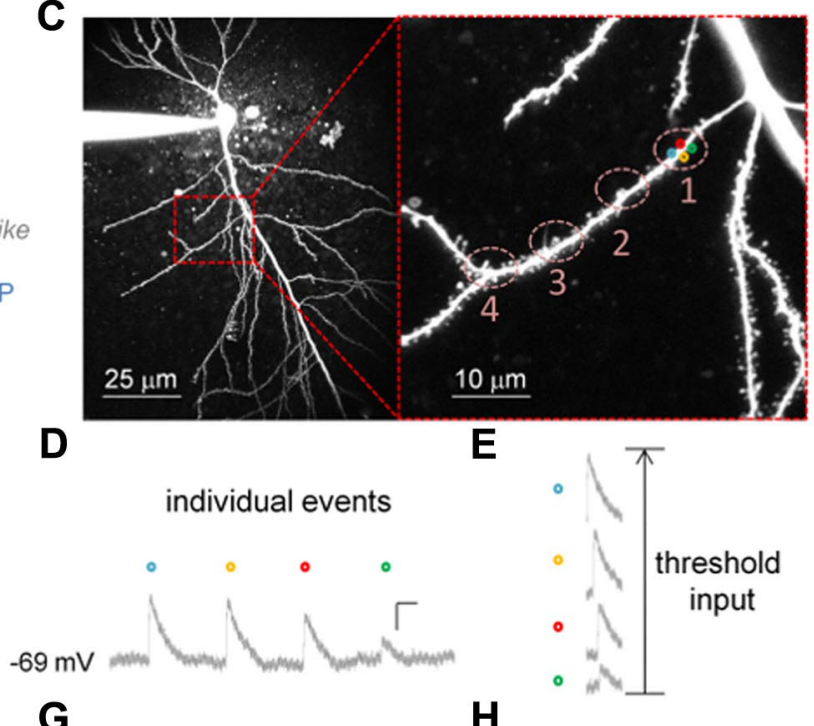

E

with the distance of the input from the bifurcation (Fig. 1G). Moreover, coupling diminished with distance from the bifurcation as indicated by both the decreasing mean ratio between $\mathrm{d}$-spike and AP thresholds and the increasing percentage of uncoupled branches (Fig. $1 H$ ).

These results show that relatively small differences in $d_{\text {dend }} / d_{\text {trunk }}$, well within the observed range of morphological diversity in the apical trees of CA1 pyramidal neurons, could have dramatic and opposing effects on the thresholds for dendritic spike initiation and propagation.

\section{Experimental validation of the computational model}

To test the physiological plausibility of the simulation results, we designed two experiments that are both relevant to the phenomena under consideration and practically achievable with available technology. First, we investigated the conditions to observe dendritic uncoupling in the slice preparation, i.e., a d-spike in the absence of somatic AP. Next, we experimentally characterized the threshold input for somatic spikes at various synaptic distances along the oblique dendrite from the bifurcation with the main trunk. In both cases, we showed that the computational model used in this study qualitatively and quantitatively reproduces the empirical findings.

A separation of $\theta_{\text {d-spike }}$ and $\theta_{\mathrm{AP}}$ is generally not observed in primary branches of CA1 pyramidal neurons: if a dendritic stimulation is sufficient to initiate a local $\mathrm{Na}^{+}$ spike, it typically evokes a somatic AP. However, these experiments seldom target the thinner obliques at farther distances from the branch point, i.e., the conditions in which threshold separation is predicted by the computational model. We hypothesized that a moderate somatic hyperpolarization could separate $\theta_{\text {d-spike }}$ and $\theta_{\mathrm{AP}}$ even for average or thicker branches stimulated closer to the bifurcation with main trunk. Using

Next, we fixed the synaptic location on the oblique branch (at 38 $\mu \mathrm{m}$ from the bifurcation) and examined the influence of using values for $d_{\text {trunk }}$ and $d_{\text {dend }}$ within the measured experimental range. Increase in trunk diameter resulted in a large rise in $\theta_{\mathrm{AP}}$ but had negligible consequences for $\theta_{\mathrm{d} \text {-spike, }}$ with uncoupling at $d_{\text {trunk }}>2.7 \mu \mathrm{m}$ (Fig. 1D). In contrast, $\theta_{\text {d-spike }}$ increased with the oblique diameter, with full coupling at $d_{\text {dend }}>0.6 \mu \mathrm{m}$ (Fig. $1 E$ ); in thinner branches, $\theta_{\mathrm{AP}}$ steeply increased as $d_{\mathrm{dend}}$ decreased and was 20 times larger than $\theta_{\mathrm{d} \text {-spike }}$ at $d_{\mathrm{dend}}=0.35 \mu \mathrm{m}$.

We also computed the initiation and propagation thresholds at a fixed distance from the bifurcation using the experimentally measured values of oblique and trunk diameters (Fig. 1E, inset). From these data, we determined the oblique/trunk diameter ratio that best discriminates between coupled and uncoupled branches (Fig. $1 F$ ). The optimally discriminating diameter ratio increased two-photon glutamate uncaging, a small cluster of synapses was stimulated with progressively higher laser powers in a fixed location on the oblique dendrite. At a holding potential $\sim 4 \mathrm{mV}$ below resting, d-spikes were clearly visible (Fig. $2 A$ ). When the geometric parameters recorded from this experiment were used in the computational model $\left(d_{\mathrm{dend}}=\right.$ $0.65 \mu \mathrm{m}, d_{\text {trunk }}=2.7 \mu \mathrm{m}$, distance from branch point of $\left.30 \mu \mathrm{m}\right)$, simulations confirmed that the oblique dendrite would be fully coupled at or above $-66 \mathrm{mV}$ but that threshold separation would be observable at or below $-67 \mathrm{mV}$ (Fig. 2B). Moreover, the simulated EPSPs, d-spikes, and APs were consistent with the experimental traces (Fig. 2A).

Dendritic filtering is affected by a combination of passive, active, and morphological characteristics, which together determine the synaptic input strength required to generate d-spikes and APs under normal conditions. A second series of experiments investigated how 

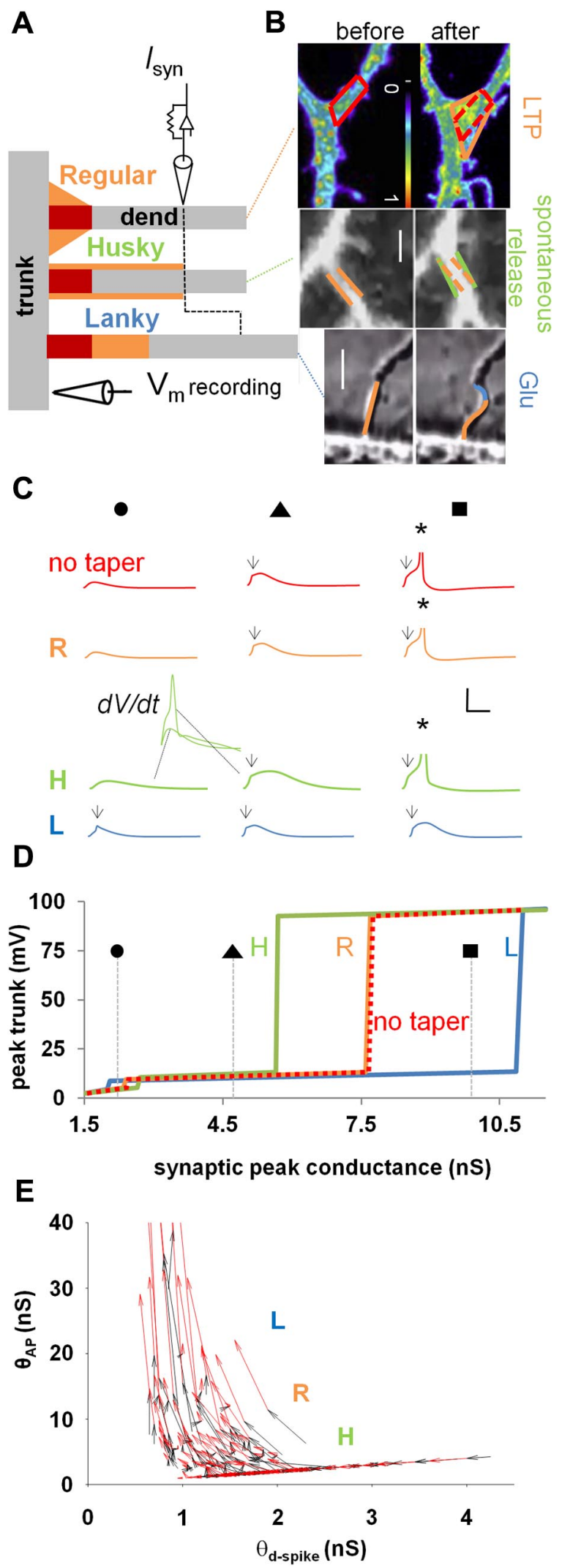

Figure 3. Potential functional effects of bifurcation morphological dynamics. $A$, Top, Regular, typical morphology. Middle, In the husky morphology, the tapering surface is redistributed to increase the dendritic diameter from the branch point up to the synaptic location. Bottom, In the lanky morphology, the tapering surface is used to increase the distance of the synaptic input from the branch point. In all cases, red indicates the membrane portion without a tapering surface; gray and black symbols schematically show the location of the synaptic input on the the somatically recorded threshold input varied with stimulus locations (Fig. $2 B-H$ ). The experimental approach was to synchronously uncage glutamate in a group of spines (Fig. $2 B$ ) and increase the intensity of each laser beam to evoke a d-spike or an AP (Fig. 2C). To circumvent the nonlinearity involved in synaptic spatial/temporal summation, the input threshold was calculated as the arithmetic sum of the unitary events, obtained by stimulating the spines asynchronously, with a 210 interval from each other (Fig. 2D,E) (Losonczy and Magee, 2006). With this protocol, small clusters of synapses (three to five) were stimulated in different obliques (Fig. 2F, different colors) at varying distances from the branch point (Fig. $2 F$, same-colored circles). The decrease of threshold input with distance could be well described in all cases with exponential functions. With the model, we then simulated this experimental design, stochastically sampling three synaptic locations in the oblique dendrite in a representatively large number of trials. The resulting simulations also demonstrated an exponential decrease of threshold input with distance (Fig. 2G). The distributions of both decay and multiplicative fitting parameters were fully consistent between model and experiments (Fig. $2 \mathrm{H}$ ) and showed no significant difference in either the Mann-Whitney or Student's $t$ tests $(p>0.1)$.

These results support the physiological plausibility of the computational model, encouraging a deeper investigation on the potential functional consequences of the observed morphological diversity at apical branch points.

\section{Functional effects of structural plasticity at branch points}

Experience can modulate a branch ability to convey signals to the rest of the neuron (Makara et al., 2009) via alterations in synaptic conductances (Tyler et al., 2007), ion channel properties (Sun, 2009), and spine morphology (Tropea et al., 2010). Our results suggest that local adjustments in branch-point morphology might also play a role in this computation. However, nothing is known about the extent and dynamics of morphological plasticity at the bifurcation between the apical trunk and oblique branches in CA1 pyramidal neurons. We re-examined the available experimental evidence originally acquired to investigate spine growth (Park et al., 2006) and filopodial sprouting (Smith and Jahr, 1992). Video captures (http:// www.sciencedirect.com/science/article/pii/S0896627306008294\# mmc6 and http://smithlab.stanford.edu/Smithlab/Dendro_Filopod_ Movies.html) demonstrate changes in both shape and size of branch

\footnotetext{
dendrite and of the recording electrode in the trunk, respectively. $\boldsymbol{B}$, Top, Branch-point exocytosis of transferrin receptor (TfR)-pHluorin after glycine-induced LTP in rat CA1 pyramidal neuron. TfR-pHluorin fluorescence was imaged before or $20 \mathrm{~min}$ after glycine stimulation. The bar $(10 \mu \mathrm{m})$ is a pseudocolor intensity scale. Image reproduced with permission from Park et al. (2006). Orange and red lines highlight the contour of the branch-point tapering region before (left, no taper) and after (right, regular) LTP induction. Middle, Imaging of CA1 pyramida neuron dendrites during spontaneous activation at time 0 (left) and 60 (right) min. Green and orange lines show a regular-to-husky transformation. Scale bar, $1 \mu \mathrm{m}$. Bottom, Response of a rat CA1 pyramidal neuron in a cell culture to a pulse of glutamate applied from a micropipette. Orange and blue lines depict the transformation from regular to lanky. Scale bar, $5 \mu \mathrm{m}$. Middle and bottom images are snapshots from the videos described by Smith and Jahr (1992), courtesy of Dr. S. Smith (Stanford University, Palo Alto, CA). C, Trunk voltage traces for four tapering shapes (without tapering membrane in red, regular in orange, husky in green, lanky in blue). Insets in middle plots represent trunk dV/dt in the absence or presence of a local spike. Symbols on top represent specific synaptic strengths, indicated in $\boldsymbol{D}$. Arrows and asterisks indicate $d$-spike and AP, respectively. $\boldsymbol{D}$, Trunk peak voltage as a function of synaptic peak conductance shows the compartmentalization associated with husky $(\mathrm{H})$, regular $(\mathrm{R})$, and lanky $(\mathrm{L})$ branch points. $\boldsymbol{E}$, Effect of changing branch-point morphology on $\theta_{\text {d-spike }}$ and $\theta_{A P}$. Black arrows show husky-to-regular changes; red arrows depict regular-to-lanky effects. For the simulations of the branch point illustrated in this figure, the synaptic input was at $38 \mu \mathrm{m}$ from the branch point for the regular and husky configurations and at $42.51 \pm 5.25 \mu \mathrm{m}$ (mean \pm SD) for the lanky case.
}

$\leftarrow$ 
points during spontaneous activation, glutamate stimulation, or induction of long-term potentiation (Fig. 3B). This evidence raises the possibility that the observed morphological diversity of branch points could result from activity-mediated modifications.

To what degree might the structural plasticity in a limited neighborhood of the branch point affect dendritic and somatic spikes? We tested computationally the potential functional effect of a modest local reorganization of the bifurcation membrane. In particular, we started from an initial "regular" shape of the branch point between the main apical trunk and oblique branch (Fig. $3 A$, top, orange plus red; compare with the post-LTP case in $B)$, with geometric parameters corresponding to the average measured values (Fig. $1 B$ ). Then we systematically manipulated a fraction of the measured tapering surface area (the orange portion, henceforth referred to as the "fringe" membrane) in different ways: (1) no taper, eliminating the fringe membrane [Fig. 3A, top branch red only (no orange); compare with the pre-LTP case in $B$ ]; (2) husky shape, obtained by redistributing the fringe membrane to thicken (on average $\sim 0.1 \mu \mathrm{m}$ for synaptic stimuli located at $50 \mu \mathrm{m}$ from the branch point) the oblique branch up to the stimulus location (Fig. $3 A$, middle branch, orange plus red; compare with the $t=60 \mathrm{~min}$ spontaneous activation case in $B$ ); and ( 3 ) lanky shape, obtained by redistributing the fringe membrane to elongate the oblique, increasing the path distance (on average $\sim 4.7 \mu \mathrm{m}$ ) between the synapse and the bifurcation (Fig. $3 A$, bottom branch, compare with the after glutamate case in $B$ ).

For each of the four conditions (regular, no taper, husky, and lanky), we determined the $\theta_{\mathrm{d} \text {-spike }}$ and $\theta_{\mathrm{AP}}$ thresholds, as in Figure $1 C-E$. Rearrangement on the fringe membrane (from regular to husky or lanky) strongly influenced both local and propagated responses in complementary and opposite directions. Specifically, stimulation intensities below $\theta_{\text {d-spike }}$ threshold for regular dendrites could often elicit a d-spike in lanky, but never in husky, dendrites (Fig. 3C, left), as also confirmed by trunk voltage derivatives (Fig. $3 C$, green inset). Conversely, stimuli above $\theta_{\mathrm{AP}}$ threshold in regular dendrites would always initiate somatic APs in husky dendrites but, in many cases, failed to do so in lanky branches (Fig. 3C, right). More generally, husky dendrites had a considerably higher threshold for dendritic spikes than regular branches but a considerably lower threshold for somatic spikes, whereas the exact opposite occurred in lanky dendrites (Fig. 3D). As a result, the range of stimulus intensities that would elicit a d-spike but not a somatic AP was twice as broad in lanky and twice as narrow in husky dendrites compared with regular branches.

Surprisingly, the presence itself of the fringe membrane never made any difference in trunk voltage and/or spike initiation and propagation thresholds, between the regular (tapering) and no taper configurations (Fig. 3C,D, orange and red traces). In other words, the amounts of input necessary to trigger dendritic or somatic spikes was not affected by the size of the tapering membrane (regular vs no taper) but by its local redistribution (husky vs lanky).

Thus, limited alterations in branch-point morphology allow for substantial bidirectional changes in the synaptic thresholds necessary to generate a local d-spike and to propagate it to the soma (Fig. 3E). Such a modest structural rearrangement could result from the local shift of an amount of membrane corresponding to the average measured fringe tapering surface, which is well within the observed variability in bifurcation morphology. This mechanism might allow neurons to greatly increase the cou-
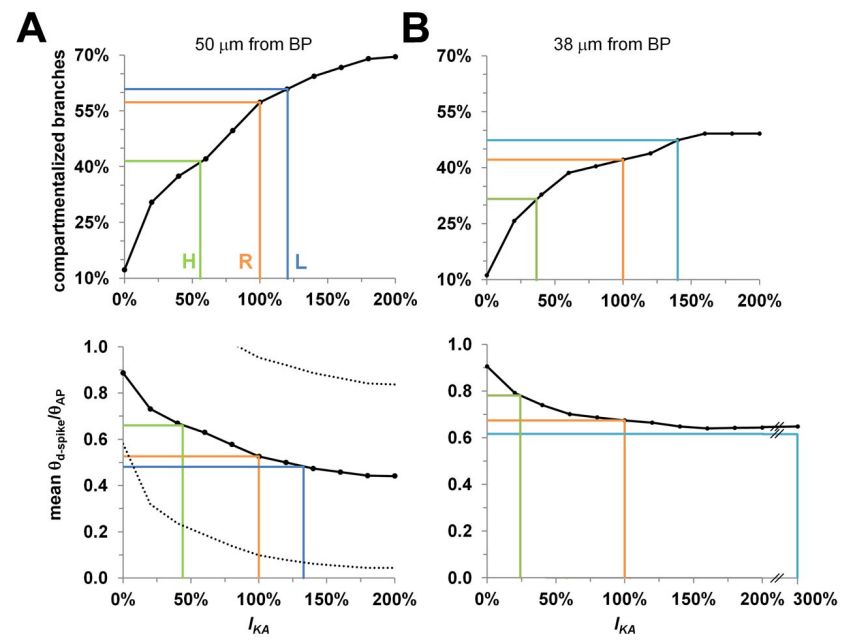

Figure 4. Functional effects of $I_{\mathrm{KA}}$ relative to variation in branch-point morphology. $\boldsymbol{A}$ Top, Fraction of compartmentalized branches as a function of $I_{\mathrm{KA}}$ activation in the regular case, with synapses distributed at $50 \mu \mathrm{m}$ from the branch point (BP). The orange line indicates the compartmentalized fraction with baseline $I_{\mathrm{KA}}$ activation. The horizontal green and blue lines mark the compartmentalized fraction in the same conditions for husky $(\mathrm{H})$ and lanky $(\mathrm{L})$ configurations, respectively. The vertical green and blue lines denote the levels of $I_{\mathrm{KA}}$ activation that yield the same compartmentalization in the regular (R) case. Bottom, Average amount of compartmentalization for uncoupled branches $\left(\theta_{\mathrm{d} \text {-spike }} /\right.$ $\theta_{\mathrm{AP}}<1$ ) as a function of $I_{\mathrm{KA}}$. The green and blue lines indicate the level of $I_{\mathrm{KA}}$ activation in the regular case that yields the same compartmentalization amount as baseline $I_{K A}$ in the husky and lanky configurations, respectively, as in $A$. Dotted lines denote SD of compartmentalization amounts. $\boldsymbol{B}$, Same as $\boldsymbol{A}$, but synaptic distance from the branch point in the regular case was $38 \mu \mathrm{m}$ instead of $50 \mu \mathrm{m}$.

pling of synapses to the trunk or else to isolate a branch from the rest of the neuron.

\section{Regulation of dendritic and somatic spiking by branch-point morphology in relation to $I_{\mathrm{KA}}$}

The A-type $\mathrm{K}^{+}$conductance $\left(I_{\mathrm{KA}}\right)$ is strongly regulated in the oblique dendrites of CA1 pyramidal neurons (Shah et al., 2010; Sun et al., 2011), providing a powerful mechanism to compartmentalize the oblique branches (Frick et al., 2003; Migliore et al., 2005). We compared the influence of structural plasticity at branch points on dendritic and somatic spike thresholds with the effect of $I_{\mathrm{KA}}$ blockage or overexpression. $I_{\mathrm{KA}}$ was regulated $(0-$ $200 \%$ ) in all the branches, and synaptic stimulation was placed at $50 \mu \mathrm{m}$ from the branch point. Additionally, we looked at the number of compartmentalized branches and at the average amount of compartmentalization for the regular, the husky, and the lanky cases, to see how they compared with $I_{\mathrm{KA}}$ modulation. In control conditions ( $100 \% I_{\mathrm{KA}}$, regular branches), the proportion of branches displaying lower thresholds for initiation than for propagation of a local spike $\left(\theta_{\mathrm{d} \text {-spike }}<\theta_{\mathrm{AP}}\right)$ depends on the distance from the bifurcation (Fig. $1 H$ ). With a stimulus $50 \mu \mathrm{m}$ from the branch point, $\sim 55 \%$ of the oblique dendrites were compartmentalized, and $45 \%$ were fully coupled with the trunk. In this condition, the average threshold ratio for d-spikes and somatic APs in the compartmentalized branches was $\sim 0.5$. Completely blocking $I_{\mathrm{KA}}$ reduced the fraction of compartmentalized branches to $12 \%$, and these had a threshold ratio close to 0.9 . In contrast, doubling $I_{\mathrm{KA}}$ increased the fraction of compartmentalized branches to $\sim 70 \%$, and these had a threshold ratio close to 0.44 (Fig. 4A). A regular-to-husky morphological transformation corresponded functionally to almost halving the distribution of dendritic $I_{\mathrm{KA}}$, whereas going from regular to lanky was equivalent 
to a $\sim 20 \% I_{\text {KA }}$ increase (Fig. $4 A$ ). Additionally, the effect of branch-point transformation was even more substantial for closer input. At $38 \mu \mathrm{m}$ from the branch point, even a $300 \%$ increase in $I_{\mathrm{KA}}$ was insufficient to match a regular-to-lanky change in average dendritic compartmentalization (Fig. 4B).

Therefore, the effect of branch-point morphological plasticity is of the same order of magnitude as that caused by alterations of active conductances. These results raise intriguing alternative hypotheses on the possible functional contribution of these two distinct mechanisms ( $I_{\mathrm{KA}}$ and branch-point morphology) in controlling branch compartmentalization. Specifically, $I_{\mathrm{KA}}$ regulation and structural plasticity might compensate each other homeostatically, they might operate independently, or their actions might be coordinated synergistically.

\section{Branch-point morphology affects delay and jitter of AP onset}

The delay between dendritic stimuli and AP onset may affect the phase of signal relative to hippocampal rhythms. Thus, we investigated whether the observed morphological diversity of oblique branches could influence AP onset in response to threshold stimulation. For regular branches, AP onset ranged over 3.5-fold, with a jitter (SD) approximately equivalent to one-third of the average value $(9.4 \pm 3.1 \mathrm{~ms}$; Fig. $5 A)$. Transforming branch points into husky and lanky morphologies reduced and increased AP onset, respectively. On average, APs in lanky dendrites were $15 \%$ slower than in husky (10.2 vs 8.9 $\mathrm{ms})$. The differences in AP onset between regular, husky, and lanky dendrites were all highly significant $\left(p<10^{-5}\right.$, onetailed paired $t$ test). Additionally, APs generated by lanky dendrites were on average $60 \%$ more jittery ( $4 \mathrm{~ms}$ ) than by husky branches (2.6 ms).

The $d_{\text {dend }} / d_{\text {trunk }}$ diameter ratio was the most relevant morphological feature affecting AP onset (Fig. 5B). Larger diameter ratios, corresponding to smaller mismatch between oblique and trunk, resulted in earlier onset. Interestingly, diameter ratios close to average (0.265) minimized AP onset jitter, whereas larger and smaller values increased jitter by several fold, from 0.38 up to $2.75 \mathrm{~ms}$ (Fig. 5B, inset). AP onset had a negative correlation with the d-spike threshold (Fig. $5 C$ ) and a strongly significant positive correlation with the AP threshold (Fig. 5D).

\section{Functional effects of branch-point morphology on an anatomically and biophysically realistic model}

The reduced model does not faithfully reproduce capacitive load of the soma and axon initial segment, as well as of the complex dendritic arbors of hippocampal neurons. To test whether these factors might affect the conclusions of this study, we replicated our most significant simulations using an existing anatomically and biophysically realistic model of a hippocampal CA1 pyramidal neuron (Ferrante et al., 2008; ModelDB accession number 119283). Specifically, we followed the same simulation protocol as in the reduced model, performing somatic recording while stimulating a specific proximal oblique dendrite (Fig. 6A). Compared with the reduced model, the selected branch demonstrated an even greater level of compartmentalization, which started at $15 \mu \mathrm{m}$ from the branch point and at $35 \mu \mathrm{m}$ reached a $\theta_{\mathrm{d} \text {-spike }} / \theta_{\mathrm{AP}}$ threshold ratio of 0.15 (Fig. $6 B$ ). Similar to the experiments and the trunk recordings in the reduced model, the amplitude of the somatic signal decayed exponentially with the synaptic distance from the branch point (Fig. $6 C$ ). Increasing the power of the stimulation (synaptic strength) evoked, in order, EPSPs, d-spikes, and APs (Fig. 6C, inset).

Next we tested the functional effects of branch-point transformations to husky or lanky morphologies for a synapse located in a region of the selected dendrite $(15.5 \mu \mathrm{m})$ that was moderately compartmentalized $\left(\theta_{\mathrm{d} \text {-spike }} / \theta_{\mathrm{AP}}=0.79\right)$ in the regular (original) morphology. The husky transformation made these synapses fully coupled with the soma $\left(\theta_{\mathrm{d} \text {-spike }} / \theta_{\mathrm{AP}}=1\right)$, whereas the lanky configuration increased compartmentalization by $36 \%\left(\theta_{\mathrm{d} \text {-spike }} /\right.$ $\left.\theta_{\mathrm{AP}}=0.58\right)$ compared with the regular branch (Fig. $6 D, E$ ). As in the reduced model, lanky dendrites had strongly delayed AP onsets with respect to husky dendrites $(\sim 13$ vs $\sim 6.5 \mathrm{~ms}$, respectively; Fig. $6 D$, inset).

Last, as in the reduced model, we modulated the $I_{\mathrm{KA}}$ distribution from 0 to $200 \%$ to compare the resulting effect on dendritic compartmentalization with that obtained by morphological change at the branch point. Completely blocking $I_{\mathrm{KA}}(0 \%)$ made the synapses fully coupled with the rest of the neuron, having the same functional effect of a husky branch. Conversely, for this specific synaptic location, there was no increase in $I_{\mathrm{KA}}$ that could reproduce the functional effect of a lanky dendrite $\left(\theta_{\mathrm{d} \text {-spike }} / \theta_{\mathrm{AP}}=\right.$ $0.58)$. The maximum compartmentalization $\left(\theta_{\mathrm{d} \text {-spike }} / \theta_{\mathrm{AP}}=0.78\right)$ was obtained by increasing $I_{\mathrm{KA}}$ to $150 \%$, which remained very close to the regular case $\left(\theta_{\mathrm{d} \text {-spike }} / \theta_{\mathrm{AP}}=0.79\right)$.

Together, these results suggest that the somatic capacitance and dendritic arborization of real neurons may amplify the effects predicted by the reduced model.

\section{Discussion}

To the best of our knowledge, this report provides the first quantification of the considerable morphological diversity of dendritic bifurcations between the apical trunk and the oblique branches in CA1 pyramidal neurons. The key result of this study is that such 
A

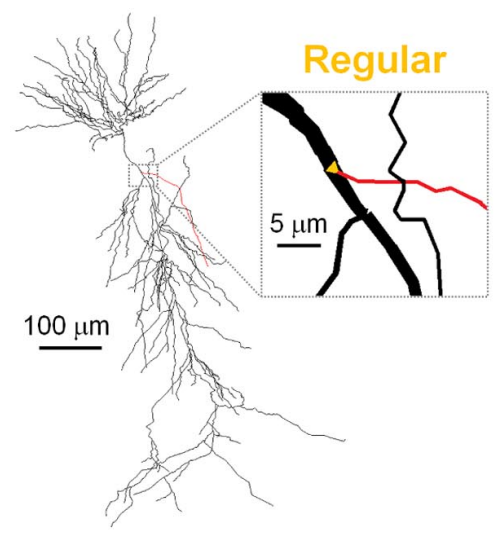

D

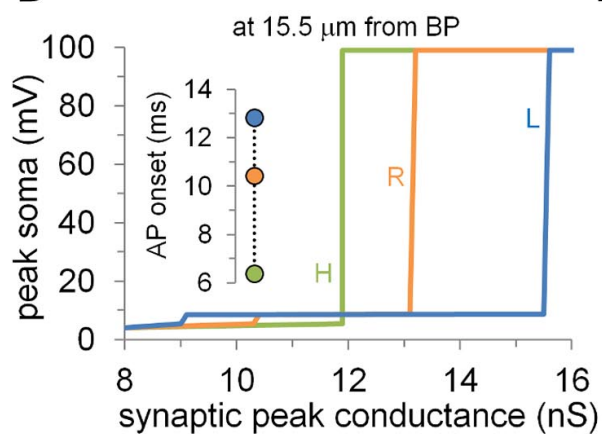

B

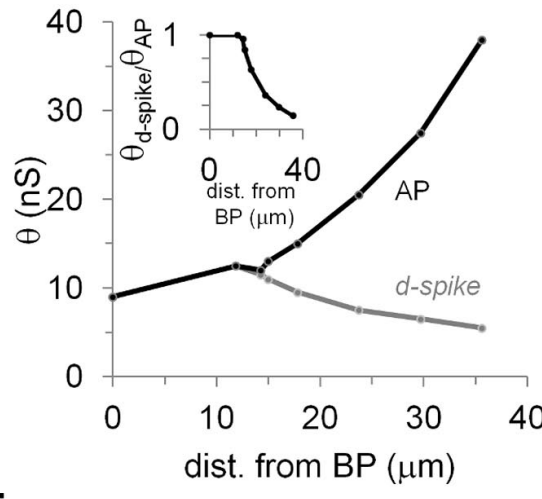

E

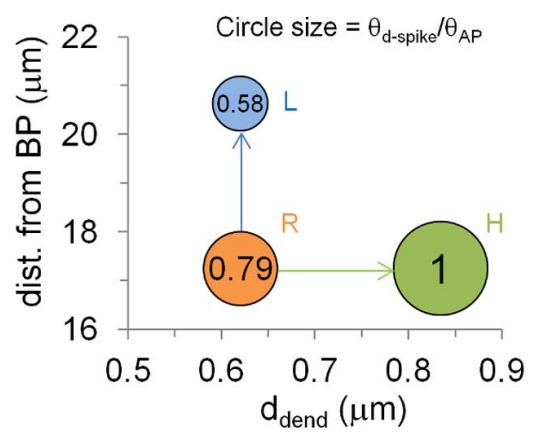

C

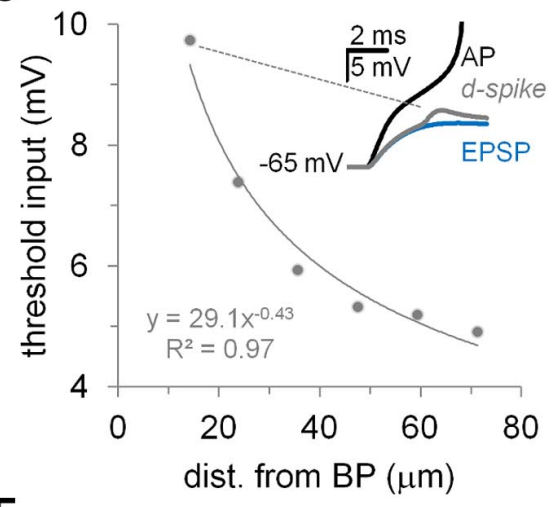

F

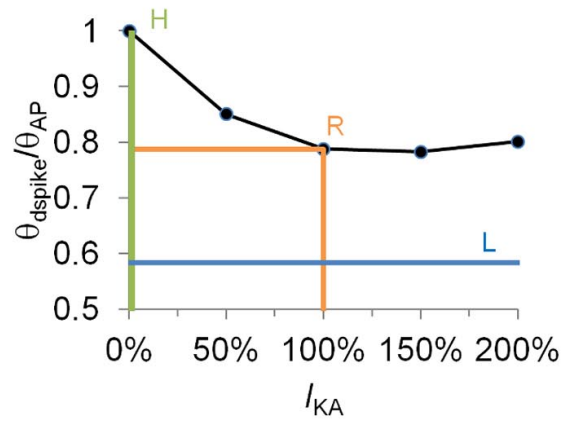

Figure 6. Effects of branch-point morphology on a realistic neuronal model. $\boldsymbol{A}$, Reconstructed soma and dendritic arborizations of a hippocampal CA1 pyramidal neuron (Ferrante et al., 2008; ModelDB accession number 119283). Inset shows magnification of the branch-point area and the oblique dendrite selected for all simulations. The branch-point morphological parameters were set to the averages of the experimentally recorded values (Fig. 1B). B , Stimulation threshold ( $\theta$ ) to generate a local dendritic spike (d-spike, gray line) or a somatic AP (black line) as a function of the distance of the input from the branch point (BP; compare with Fig. 1C). Inset depicts $\theta_{\mathrm{d} \text {-spike }} / \theta_{\mathrm{AP}}$ threshold ratio as a function of the distance of the input from the branch point (compare with Fig. $1 H)$. C, Exponential decay of the computed threshold input as a function of the distance of the synaptic input from the branch point (compare with Fig. 2C,D). Inset, Gray voltage trace shows the d-spike for the specific computed threshold input; AP (black line) and voltage signatures for the same synaptic cluster are also shown (compare with Fig. $2 A$ ). $D$, Somatic peak voltage as a function of synaptic peak conductance shows the compartmentalization associated with husky ( $H$, green), regular ( $R$, orange), and lanky ( $L$, green) branch points (compare with Fig. 3D). Inset, $A P$ onset time for the three morphological configurations: husky, regular, and lanky. $\boldsymbol{E}$, Symbol size represents $\theta_{\mathrm{d} \text {-spike }} / \theta_{\mathrm{AP}}$ threshold ratio as a function of $d_{\text {dend }}$ and synaptic distance from the branch point for the husky, regular, and lanky branch shapes (compare with Figs. $1 F, 7 A)$. $\boldsymbol{F}$, Amount of compartmentalization $\left(\theta_{\text {d-spike }} / \theta_{\text {AP }}<1\right)$ as a function of $I_{\text {KA }}$ activation. The orange line indicates the regular branch compartmentalization with baseline $I_{\mathrm{KA}}$ activation. The green and blue lines mark the branch compartmentalization corresponding to the husky and lanky configurations, respectively (compare with Fig. 4C,D).

diversity may significantly affect the coupling of synaptic inputs to somatic output. Specifically, computational simulations prove that the observed variability of branch morphology yields a broad range of functional compartmentalization. This phenomenology can be summarized in a surface plot (Fig. $7 A$ ) in which the oblique diameter and the distance of the stimulus from the bifurcation are the independent variables and the local and propagated spike threshold(s) are the dependent variable(s). Relevant experiments (Fig. 2) indicate that the biophysical parameters of the model are adequate to reproduce empirical electrophysiological recordings.

\section{Fringe tapering membrane as a currency for compartmentalization}

Available evidence from time-lapse imaging indicates that oblique branches of CA1 pyramidal neurons can change their shape and size in a matter of minutes under normal conditions. Thus, we hypothesized that branch-point morphology could dynamically adapt over a husky-lanky continuum (Smith and Jahr, 1992; Park et al., 2006). Such transitions effectively constitute movements along the independent axes of the above-described surface plot, reflected by functional consequences along the dependent axis. Within realistic parameter ranges (measured oblique diameter of $0.33-0.75 \mu \mathrm{m}$, reasonable path distance of the stimulus from the bifurcation of $0-70 \mu \mathrm{m})$, the local spiking threshold ranges from $\sim 0.1$ to $\sim 10 \mathrm{nS}$. The low and high ends of this span approximately correspond to activating a single excitatory synapse or (quasi-synchronously) all the excitatory synapses on the oblique branch.

This mechanism has been neglected in experiments so far, but, surprisingly, our model predicts that a local rearrangement of the fringe tapering membrane can switch virtually any oblique branch between fully coupled $\left(\theta_{\mathrm{d} \text {-spike }}=\theta_{\mathrm{AP}}\right)$ and strongly compartmentalized $\left(\theta_{\mathrm{d} \text {-spike }} \ll \theta_{\mathrm{AP}}\right)$. The "typical" branch, corresponding to the average experimental measurements (Fig. $7 \mathrm{~A}$, red dot), is moderately compartmentalized in the regular tapering shape $\left(\theta_{\mathrm{AP}} \approx 2 \times \theta_{\mathrm{d} \text {-spike }}\right.$ for stimuli at $45 \mu \mathrm{m}$ from the branch point). Morphing the fringe membrane to a lanky shape (blue dot) halves the d-spike threshold and doubles the AP threshold (higher compartmentalization). Conversely, changing to a husky shape (green dot) causes the two thresholds to converge to closer values (higher coupling). This suggests that the bifurcation tapering membrane can be a quintessential determinant of local and global excitability.

To generalize and quantify this conclusion, it is useful to compute the extent of the functionally relevant alterations (path distance and dendritic diameter) per unit of rearranged fringe membrane (Fig. $7 B$ ). Clearly, it takes more membrane area to 
A

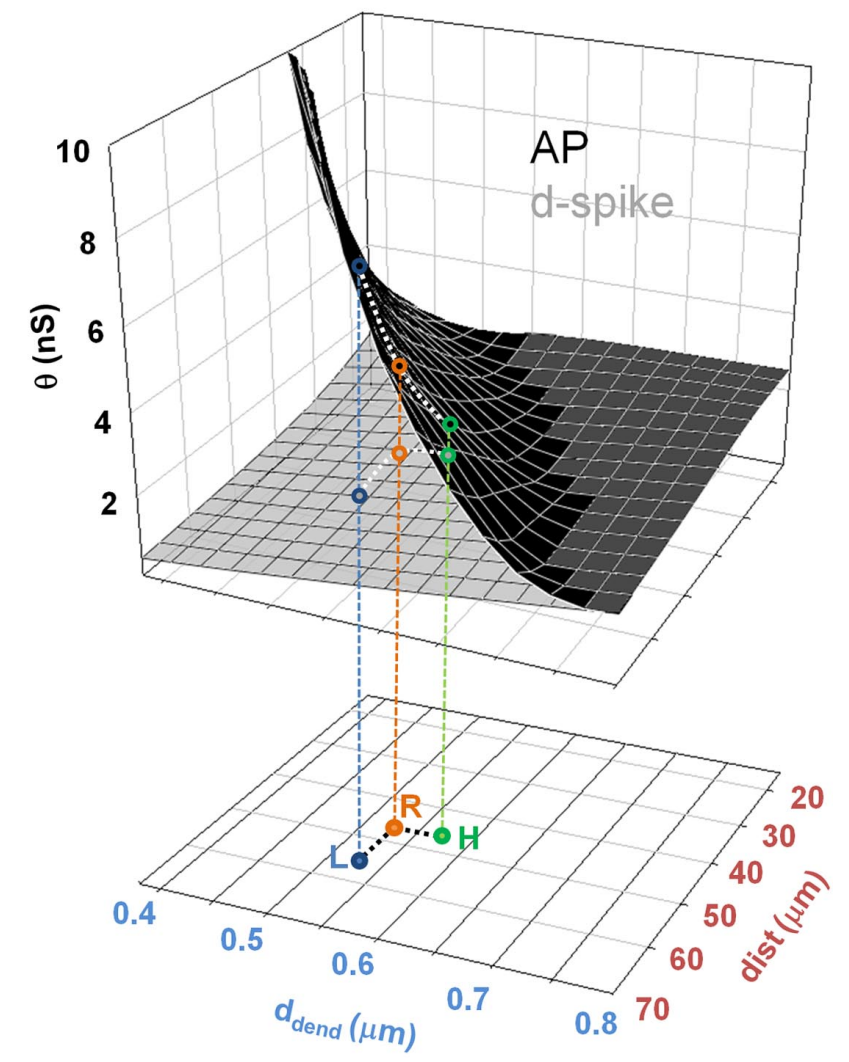

B

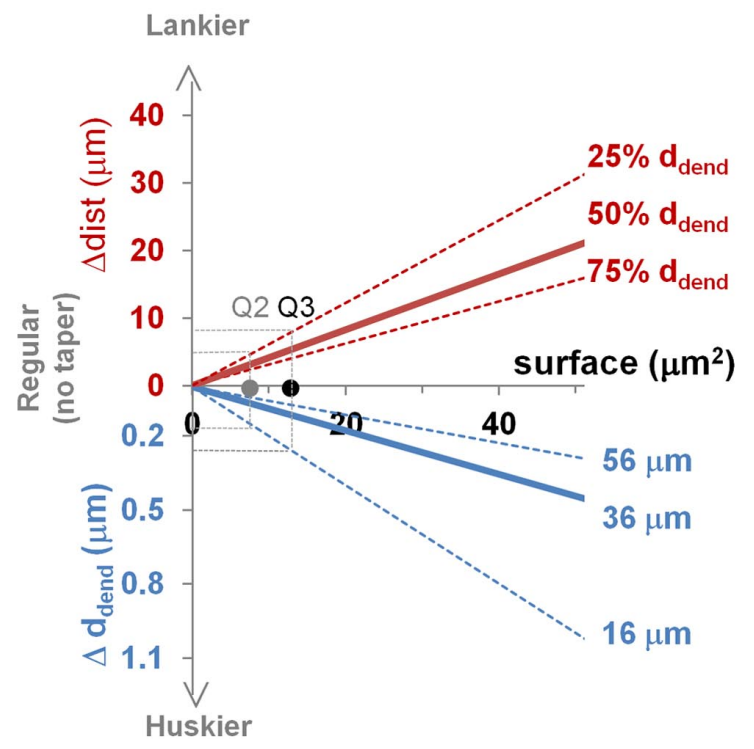

Figure 7. Functional consequences of changing branch-point morphology. $\boldsymbol{A}$, Top, Threshold separation ( $\theta_{\text {d-spike, }}$ gray; $\theta_{\mathrm{AP}}$, black), as a function of the oblique branch diameter and of the distance of the synaptic input from the branch point; the white dotted lines indicate the threshold change with branch-point morphology. Bottom, Representative parameters for a regular branch ( $\mathrm{R}$, orange dot) and the corresponding husky $(\mathrm{H}$, green dot) and lanky (L, blue dot) branches obtained by reconfiguring the fringe taper membrane. The vertical projections (orange, blue, and green lines) indicate the corresponding threshold separation effects. $\boldsymbol{B}$, Top (red), Amount of elongation obtained by reconfiguring the fringe membrane from regular to lanky as a function of the taper surface area at the first, median, and third quartiles of oblique branch diameter. Bottom (blue), Amount of thickening obtained by reconfiguring the fringe membrane from regular to husky as a function of the branch-point surface for a three stimulation distances from the bifurcation. Gray and black dots and dotted lines denote the effects for the median (Q2) and third (Q3) quartiles of the measured surface distribution, respectively. elongate thicker than thinner branches. Similarly, more membrane area is required to thicken the dendritic path to distal compared with proximal stimulus locations. Notably, the median measured fringe membrane area is sufficient to yield dramatic changes across the bottom, median, and top quartiles of oblique dendritic diameters and through a wide span of distances along the branch path. Thus, the fringe tapering membrane at branch point could act as currency to bidirectionally exchange for synaptic compartmentalization or coupling.

The lower threshold for dendritic spikes would enable compartmentalized branches to express higher local activity without affecting overall synaptic integration and signal processing in the rest of the neuron. Periods of increased local firing may facilitate the rapid adjustment of synaptic conductances and other activity-dependent plastic processes.

\section{Branch-point morphology and other determinants of the input/output relationship}

Our computational simulations predict that minor structural differences at the branch point can produce major alterations in the integrative properties of oblique dendrites. Independent of whether such structural differences might dynamically result from experience-dependent plasticity, they are well within the observed range of natural variability. The ensuing functional changes in local and propagated spiking thresholds are comparable in magnitude with those produced by modulation of active properties, such as $I_{\mathrm{KA}}$. Thus, morphological differences between branches might reverse or accentuate the local regulation of voltage-gated conductances. The recently demonstrated selective exocytosis of post-Golgi cargo at branch points (Cui-Wang et al., 2012) may provide the mechanism for continuous control over membrane addition. In CA1 pyramidal neurons, $I_{\mathrm{KA}}$ has recently been involved in synaptic scaling (Ito and Schuman, 2009), and Kv1.1 and Kv1.4 knockdown ablate LTP (Meiri et al., 1998). Branch-point morphology thus adds a third factor in this interaction. Because branch-point morphological plasticity can affect both local and global spike thresholds (Fig. 1) and AP timing (Fig. 5 ), we suggest that this mechanism might also play a strong role in modulating spike-timing-dependent plasticity. Additionally, although $I_{\mathrm{KA}}$ is specifically involved in compartmentalization, several other channels affect signal propagation, including $I_{\mathrm{Ca}}$ and $I_{h}$ (Magee, 1998; Cai et al., 2004; Migliore et al., 2004; Johnston and Narayanan, 2008).

In neocortical neurons, the interrelation among dendritic cross-sections at branch points may facilitate spreading depolarization (Kubota et al., 2011), and the ratio of proximal over distal oblique branches affects the coupling between somatic and dendritic spikes (Schaefer et al., 2003). It is unknown whether similar considerations apply to CA1 pyramidal neurons and whether the findings of the present study are applicable to other neuron types. How branch-point morphology affects the spatiotemporal integration of multiple synaptic signals at different input frequencies also remains to be determined. We have shown that, at threshold, husky dendrites were on average faster and more precise $(60 \%$ less jittery) than lanky branches. These results suggest that small branch-point morphological transformations might also affect the synchrony of synaptic input from different branches. It is becoming clear that the branch propagation of EPSPs and d-spikes depends on the interplay between dendritic morphology, synaptic dynamics, and membrane biophysics. 


\section{References}

Andrasfalvy BK, Magee JC (2001) Distance-dependent increase in AMPA receptor number in the dendrites of adult hippocampal CA1 pyramidal neurons. J Neurosci 21:9151-9159. Medline

Bourne JN, Harris KM (2008) Balancing structure and function at hippocampal dendritic spines. Annu Rev Neurosci 31:47-67. CrossRef Medline

Cai X, Liang CW, Muralidharan S, Kao JP, Tang CM, Thompson SM (2004) Unique roles of SK and $\mathrm{Kv} 4.2$ potassium channels in dendritic integration. Neuron 44:351-364. CrossRef Medline

Canepari M, Djurisic M, Zecevic D (2007) Dendritic signals from rat hippocampal CA1 pyramidal neurons during coincident pre- and post-synaptic activity: a combined voltage- and calcium-imaging study. J Physiol 580:463-484. CrossRef Medline

Caporale N, Dan Y (2008) Spike timing-dependent plasticity: a Hebbian learning rule. Annu Rev Neurosci 31:25-46. CrossRef Medline

Cui-Wang T, Hanus C, Cui T, Helton T, Bourne J, Watson D, Harris KM, Ehlers MD (2012) Local zones of endoplasmic reticulum complexity confine cargo in neuronal dendrites. Cell 148:309-321. CrossRef Medline

Ferrante M, Blackwell KT, Migliore M, Ascoli GA (2008) Computational models of neuronal biophysics and the characterization of potential neuropharmacological targets. Curr Med Chem 15:2456-2471. CrossRef Medline

Frick A, Magee J, Koester HJ, Migliore M, Johnston D (2003) Normalization of $\mathrm{Ca}^{2+}$ signals by small oblique dendrites of $\mathrm{CA} 1$ pyramidal neurons. J Neurosci 23:3243-3250. Medline

Fu M, Zuo Y (2011) Experience-dependent structural plasticity in the cortex. Trends Neurosci 34:177-187. CrossRef Medline

Gasparini S, Migliore M, Magee JC (2004) On the initiation and propagation of dendritic spikes in CA1 pyramidal neurons. J Neurosci 24:1104611056. CrossRef Medline

Golding NL, Spruston N (1998) Dendritic sodium spikes are variable triggers of axonal action potentials in hippocampal CA1 pyramidal neurons. Neuron 21:1189-1200. CrossRef Medline

Grunditz A, Holbro N, Tian L, Zuo Y, Oertner TG (2008) Spine neck plasticity controls postsynaptic calcium signals through electrical compartmentalization. J Neurosci 28:13457-13466. CrossRef Medline

Hines ML, Carnevale NT (1997) The NEURON simulation environment. Neural Comput 9:1179-1209. CrossRef Medline

Hoffman DA, Magee JC, Colbert CM, Johnston D (1997) K+ channel regulation of signal propagation in dendrites of hippocampal pyramidal neurons. Nature 387:869-875. CrossRef Medline

Ito HT, Schuman EM (2009) Distance-dependent homeostatic synaptic scaling mediated by a-type potassium channels. Front Cell Neurosci 3:15. CrossRef Medline

Johnston D, Narayanan R (2008) Active dendrites: colorful wings of the mysterious butterflies. Trends Neurosci 31:309-316. CrossRef Medline

Katz Y, Menon V, Nicholson DA, Geinisman Y, Kath WL, Spruston N (2009) Synapse distribution suggests a two-stage model of dendritic integration in CA1 pyramidal neurons. Neuron 63:171-177. CrossRef Medline

Komendantov AO, Ascoli GA (2009) Dendritic excitability and neuronal morphology as determinants of synaptic efficacy. J Neurophysiol 101: 1847-1866. CrossRef Medline

Krichmar JL, Nasuto SJ, Scorcioni R, Washington SD, Ascoli GA (2002) Effects of dendritic morphology on CA3 pyramidal cell electrophysiology: a simulation study. Brain Res 941:11-28. CrossRef Medline

Kubota Y, Karube F, Nomura M, Gulledge AT, Mochizuki A, Schertel A, Kawagchi Y (2011) Conserved properties of dendritic trees in four cortical interneuron subtypes. Sci Rep 1:89. CrossRef Medline

Li X, Ascoli GA (2006) Computational simulation of the input-output relationship in hippocampal pyramidal cells. J Comput Neurosci 21:191-209. CrossRef Medline

Losonczy A, Magee JC (2006) Integrative properties of radial oblique dendrites in hippocampal CA1 pyramidal neurons. Neuron 50:291-307. CrossRef Medline

Losonczy A, Makara JK, Magee JC (2008) Compartmentalized dendritic plasticity and input feature storage in neurons. Nature 452:436-441. CrossRef Medline

Magee JC (1998) Dendritic hyperpolarization-activated currents modify the integrative properties of hippocampal CA1 pyramidal neurons. J Neurosci 18:7613-7624. Medline

Mainen ZF, Sejnowski TJ (1996) Influence of dendritic structure on firing pattern in model neocortical neurons. Nature 382:363-366. CrossRef Medline

Makara JK, Losonczy A, Wen Q, Magee JC (2009) Experience-dependent compartmentalized dendritic plasticity in rat hippocampal CA1 pyramidal neurons. Nat Neurosci 12:1485-1487. CrossRef Medline

Megías M, Emri Z, Freund TF, Gulyás AI (2001) Total number and distribution of inhibitory and excitatory synapses on hippocampal CA1 pyramidal cells. Neuroscience 102:527-540. CrossRef Medline

Meiri N, Sun MK, Segal Z, Alkon DL (1998) Memory and long-term potentiation (LTP) dissociated: normal spatial memory despite CA1 LTP elimination with Kv1.4 antisense. Proc Natl Acad Sci U S A 95:15037-15042. CrossRef Medline

Migliore M, Messineo L, Ferrante M (2004) Dendritic $I_{h}$ selectively blocks temporal summation of unsynchronized distal inputs in CA1 pyramidal neurons. J Comput Neurosci 16:5-13. CrossRef Medline

Migliore M, Ferrante M, Ascoli GA (2005) Signal propagation in oblique dendrites of CA1 pyramidal cells. J Neurophysiol 94:4145-4155. CrossRef Medline

Morita K (2008) Possible role of dendritic compartmentalization in the spatial working memory circuit. J Neurosci 28:7699-7724. CrossRef Medline

Nolan MF, Malleret G, Dudman JT, Buhl DL, Santoro B, Gibbs E, Vronskaya S, Buzsáki G, Siegelbaum SA, Kandel ER, Morozov A (2004) A behavioral role for dendritic integration: HCN1 channels constrain spatial memory and plasticity at inputs to distal dendrites of CA1 pyramidal neurons. Cell 119 [Erratum (2005) 120:151-152]:719-732. CrossRef Medline

Park M, Salgado JM, Ostroff L, Helton TD, Robinson CG, Harris, KM, Ehlers MD (2006) Plasticity-induced growth of dendritic spines by exocytic trafficking from recycling endosomes. Neuron 52:817-830. CrossRef Medline

Poirazi P, Mel BW (2001) Impact of active dendrites and structural plasticity on the memory capacity of neural tissue. Neuron 29:779-796. CrossRef Medline

Rabinowitch I, Segev I (2006) The interplay between homeostatic synaptic plasticity and functional dendritic compartments. J Neurophysiol 96:276-283. CrossRef Medline

Remy S, Csicsvari J, Beck H (2009) Activity-dependent control of neuronal output by local and global dendritic spike attenuation. Neuron 61:906-916. CrossRef Medline

Schaefer AT, Larkum ME, Sakmann B, Roth A (2003) Coincidence detection in pyramidal neurons is tuned by their dendritic branching pattern. J Neurophysiol 89:3143-3154. CrossRef Medline

Scorcioni R, Lazarewicz MT, Ascoli GA (2004) Quantitative morphometry of hippocampal pyramidal cells: differences between anatomical classes and reconstructing laboratories. J Comp Neurol 473:177-193. CrossRef Medline

Shah MM, Hammond RS, Hoffman DA (2010) Dendritic ion channel trafficking and plasticity. Trends Neurosci 33:307-316. CrossRef Medline

Smith SJ, Jahr CE (1992) Rapid induction of filipodial sprouting by application of glutamate to hippocampal neurons. In: The nerve growth cone, Chap 3 (Letourneau PC, Kater SB, Macagno ER, eds). New York: Raven.

Sun QQ (2009) Experience-dependent intrinsic plasticity in interneurons of barrel cortex layer IV. J Neurophysiol 102:2955-2973. CrossRef Medline

Sun W, Maffie JK, Lin L, Petralia RS, Rudy B, Hoffman DA (2011) DPP6 establishes the A-type $\mathrm{K}^{+}$current gradient critical for the regulation of dendritic excitability in CA1 hippocampal neurons. Neuron 71:11021115. CrossRef Medline

Tropea D, Majewska AK, Garcia R, Sur M (2010) Structural dynamics of synapses in vivo correlate with functional changes during experiencedependent plasticity in visual cortex. J Neurosci 30:11086-11095. CrossRef Medline

Turrigiano G, Abbott LF, Marder E (1994) Activity-dependent changes in the intrinsic properties of cultured neurons. Science 264:974-977. CrossRef Medline

Tyler WJ, Petzold GC, Pal SK, Murthy VN (2007) Experience-dependent modification of primary sensory synapses in the mammalian olfactory bulb. J Neurosci 27:9427-9438. CrossRef Medline 